\title{
Reply to 'Comment on 'Effect of population breast screening on breast cancer mortality up to 2005 in England and Wales: an individual-level cohort study"
}

Stephen Morrell ${ }^{*}$, David Roder $^{2}$ and Richard Taylor $^{1}$

${ }^{1}$ Faculty of Medicine, School of Public Health and Community Medicine, University of New South Wales, Sydney 2052, NSW, Australia and ${ }^{2}$ School of Health Sciences, Centre for Population Research, Sansom Institute for Health Research, University of South Australia, Adelaide, SA 5000, Australia

Sir,

The recent communication by Miller (2017) asserts that 'As we demonstrated in Canada (Miller et al, 1992a, b, 2000, 2002, 2014), it is not possible to demonstrate a reduction in breast cancer mortality due to mammography screening in an era of modern adjuvant therapy for breast cancer.'

We contend that this characterisation of the effects of modern adjuvant therapy on breast cancer mortality in relation to screening mammography is erroneous. In the Canadian example cited by Miller, along with other screening mammography programs (including Australia), mammography screening was introduced at the same time that improvements to adjuvant therapies were occurring, in the late 1980s and 1990s. In this circumstance, it is indeed difficult to separate out the associations of screening versus therapy improvements with breast cancer mortality. However, during a period when a treatment regime undergoes few changes, whether the therapy is 'modern' or medieval, any screening-related breast cancer mortality differences would not be confounded by treatment improvements if none were occurring during the study period.

Moreover, an Australian incidence-linked study (Morrell et al, 2012), based on small areas and annual screening and breast cancer incidence data, found mammography screening exposure was significantly and negatively correlated with subsequent breast cancer mortality in these 78000 strata cohorts. The probability of adjuvant treatment (somehow) co-varying with breast cancer mortality and/or screening in these strata to confound the observed association between screening and breast cancer mortality is negligible. Similarly and more generally, in individualbased case-control or cohort studies examining associations of screening intensity with breast cancer mortality, there would need to be similar co-varying of treatment quality/effectiveness with levels of mammography exposure. Yet, the results from these studies are similar irrespective of study design and screening setting.

Miller quotes a recent article by Morrell et al (2017) in relation to screening mammography in New Zealand to bolster his case. However, screening mammography was introduced in New Zealand in 1999, well after the major improvements in adjuvant therapy. While we may have made 'complicated' adjustments for screening participation bias in this study, these had no bearing on whether our findings were confounded by variations in treatment. In the case of New Zealand, they were not.

\section{CONFLICT OF INTEREST}

The authors declare no conflict of interest.

\section{REFERENCES}

Morrell S, Taylor R, Roder D, Dobson A (2012) Mammography screening and breast cancer mortality in Australia: an aggregate cohort study. J Med Screen 19: $26-34$.

Morrell S, Taylor R, Roder D, Robson B, Gregory M, Craig K (2017)

Mammography service screening and breast cancer mortality in New Zealand: a National Cohort Study 1999-2011. Br J Cancer 116: 828-839.

Miller AB (2017) Comment on 'Effect of population breast screening on breast cancer mortality up to 2005 in England and Wales: an individual-level cohort study'. Br J Cancer 117: e2.

This work is published under the standard license to publish agreement. After 12 months the work will become freely available and the license terms will switch to a Creative Commons Attribution-onCommercial-hare Alike 4.0 Unported License. 